\title{
Decoupled, energy stable schemes for a phase-field surfactant model
}

\author{
Guangpu Zhu', Jisheng Kou ${ }^{\mathrm{b}}$, Shuyu Sun ${ }^{\mathrm{c}, *}$, Jun Yao ${ }^{\mathrm{a}, *}$ and Aifen $\mathrm{Li}^{\mathrm{a}}$ \\ ${ }^{a}$ Research Center of Multiphase Flow in Porous Media, School of Petroleum Engineering, China University of Petroleum (East \\ China), Qingdao 266580, China \\ b School of Mathematics and Statistics, Hubei Engineering University, Xiaogan 432000, Hubei, China. \\ ${ }^{c}$ Computational Transport Phenomena Laboratory, Division of Physical Science and Engineering, King Abdullah University of \\ Science and Technology, Thuwal 23955-6900, Kingdom of Saudi Arabia
}

\begin{abstract}
In this paper, we construct several linear, decoupled and energy stable schemes for a phase-field surfactant model, in which the free energy functional contains a fourth-order Ginzburg-Landau double well potential, a logarithmic Flory-Huggins potential and two nonlinear coupling terms. Several scalar auxiliary variables (SAV) are introduced to transform the governing system into an equivalent form, allowing the nonlinear potentials to be treated efficiently and semi-explicitly. At each time step, the schemes involve solving only two linear elliptic differential equations, and computations of two phase-field variables are totally decoupled. Moreover, the local concentration of surfactants can be obtained in a "explicit" way. We further establish a rigorous proof of unconditional energy stability for the semi-implicit schemes. Numerical results in both two and three dimensions are obtained, which demonstrate that the proposed schemes are accurate, efficient, easy-to-implement and unconditionally energy stable.
\end{abstract}

Keywords: Phase-field; Cahn-Hilliard; Fluid-surfactant; Scalar auxiliary variable; Energy stability

\section{Introduction}

Surfactants, interface active agents, are known to effectively reduce the interfacial tension of two immiscible fluids (e.g., the mixture oil and water), and allow for the formation of emulsions $[1,2]$. Surfactant molecules are composed of hydrophilic heads and hydrophobic tails. Due to this molecular composition, surfactants selectively adhere to fluid interface (hydrophilic heads into the water, hydrophobic tails into the oil) forming a buffer zone to reduce the system energy [3]. This particular property makes surfactants widely used in many industrial processes, including the manufacture of cosmetics, food processing and enhanced oil recovery [3, 4].

Numerous studies on modeling and numerical simulations have been conducted to investigate the binary fluid-surfactant system. There are basically two ways to simulate the interfacial dynamics with surfactants in a multiphase system. Most previous work on surfactants combined sharp interface models with the equilibrium equation of state [5-10], which relates the dynamic interfacial tension with the local surfactant concentration. Although these sharp interface models have made great progresses in simulating the multiphase system with surfactants [7, 9], they still suffer from some drawbacks [3]. The diffusive interface method, or called the phase-field method $[2,11-21]$, is the other popular tool to investigate the interfacial dynamics with surfactants [1, 22-25]. Contrast to sharp interface models, the phase-field model utilizes an appropriate free energy functional [26-28] to resolve the interfacial dynamics, and thus have a firm physical basis for multiphase flows [3]. The classical Ginzburg-Landau potential is usually used to describe a binary fluid system. For a binary fluid-surfactant system, Laradji et al. first introduced some 
additional terms to the original Ginzburg-Landau potential to account for the effect of surfactants [29]. Two phase variables were used in their model to represent the local densities of the fluids and the local concentration of surfactants, respectively. The coupling term in the proposed free energy functional globally counteracts the occurrence of free surfactants in the bulk phases, and the nonlinearly coupled entropy term locally attracts surfactants to an existing interface $[3,29]$. Since then, a variety of phase-field surfactant models have been proposed and review of these models can refer to [30-32]. Here we only highlight some representative work. Komura et at. observed that the model proposed by Laradji was numerically unstable as the simulation mesh size decreases. They proposed a different two-order-parameter Ginzburg-Landau free energy to improve the numerical stability [33]. Theissen and Gompper made a minor change to the local coupling term of Laradji's model to deal with the same solubility of surfactants in the bulk phases [34]. In [35], Samn and Graaf introduced the logarithmic Floy-Huggins potential to restrict the range of local concentration of surfactants. The above models essentially share the same nonlinearly coupled entropy term. Recently, a slightly different local coupling entropy was proposed in [36].

Due to the stiffness associated with the interface width and strong nonlinear couplings between two phase field variables [16, 37], it is a challenging work to develop some efficient and energy sable schemes for the phase field-surfactant models. Although a series of binary fluid-surfactant phase field (BFS-PF) models have been proposed, there are very few successful attempts in constructing schemes with unconditional stability for them. Gu et al. presented an energy stable finite-difference scheme for the BFS-PF model in [38]. The proposed scheme was based on the convex splitting of the energy functional with two phase variables. From the view of positivity of second-order partial derivatives, their arguments about the convex splitting strategy for the coupling potential are not valid because it is not sufficient to justify the convexity of a function with multiple variables [37]. Furthermore, the nonlinear nature of this scheme leads to high computational costs. Most recently, Yang et al. constructed a first-order and a second-order energy stable schemes for the phase-field surfactant model in [37] using the Invariant Energy Quadratization (IEQ) approach [39, 40]. With the introduction of new variables, the free energy is transformed into an equivalent quadratic form. All nonlinear terms in the transformed system are treated semi-explicitly in their schemes [37]. Their schemes are the first such linear, accurate and energy stable schemes for the nonlinear coupled multivariate model. However, the IEQ approach leads to a more complicated nonlinearly coupled terms in the chemical potential associated with the local densities of the two fluids, and the coupling of the two phase variables further increases the difficulty of solving. Therefore, the main purpose of this study is to construct some more efficient, accurate schemes with unconditional energy stability for a BFS-PF model.

To develop the desired schemes, a new numerical technique, the scalar auxiliary variable (SAV) approach [41-43], is adopted to deal with nonlinear terms. The SAV approach is built on the recently introduced IEQ approach. It enjoys all advantages of the IEQ approach but overcoming most of its shortcomings [41]. A series of recent studies found that the SAV approach may be more accurate than the IEQ and stabilization approaches at the same time step size [41, 42]. In this approach, several scalar auxiliary variables are introduced to transform the original free energy into an equivalent form, lowing the nonlinear potentials to be treated efficiently and semi-explicitly. It worth emphasizing that the developed schemes in this study can be extended to a large class of gradient flow problems with multiple variables or complex nonlinearities in the 
free energy density.

The rest of this paper is organized as follows. In section 2, we describe a nonlinearly coupled BFS-PF model, and transform this model into a new equivalent system by using the SAV approach In section 3, we construct several efficient, decoupled and energy stable schemes for the transformed system and carry out the energy stability estimate for the proposed schemes. Several 2D and 3D numerical examples are investigated in Section 4 and the paper is finally concluded in Section 5 .

\section{The governing equation}

For a multiphase system with surfactants, we consider a typical representative of nonlinearly coupled binary fluid-surfactant phase field models, which was introduced in [3, 35, 44]. The total free energy of the system reads:

$$
E_{\text {tot }}(\phi, \rho)=E_{1}(\phi)+E_{2}(\rho)+\int\left(\frac{\alpha}{2} \rho \phi^{2}-\theta \rho|\nabla \phi|^{2}\right) d \Omega,
$$

where

$$
\begin{gathered}
E_{1}(\phi)=\int\left(\frac{\lambda \varepsilon}{2}|\nabla \phi|^{2}+\lambda F(\phi)\right) d \Omega, \\
E_{2}(\rho)=\int \beta G(\rho) d \Omega, \\
F(\phi)=\frac{1}{4 \varepsilon}\left(\phi^{2}-1\right)^{2}, \quad G(\rho)=\rho \ln \rho+(1-\rho) \ln (1-\rho),
\end{gathered}
$$

here $\lambda, \beta, \alpha$, and $\theta$ are all positive parameters, $\phi$ is a phase variable,

$$
\phi(x, t)= \begin{cases}1 & \text { phase } 1, \\ -1 & \text { phase } 2 .\end{cases}
$$

$E_{1}(\phi)$ is the commonly-used Ginzburg-Landau type of Helmholtz free energy functional. The first term in $E_{1}(\phi)$ contributes to the two-phase mixing and the second part, the double well bulk energy $F(\phi)$, promotes the two-phase separation. The competition between the two types of interaction creates a diffusive interface between fluids.

$E_{2}(\rho)$ is the free energy associated with the local concentration of surfactants [36]. The other phase variable $\rho$ is introduced to represent the local concentration of surfactants. $G(\rho)$ is the logarithmic Flory-Huggins type energy potential. The first term $\rho \ln \rho$ in $G(\rho)$ models the ideal mixing of surfactants in fluids and guarantees the value of $\rho$ to be positive, and the second term $(1-\rho) \ln (1-\rho)$ restricts $\rho<1$. Note that the lower bound of $\rho$ corresponds to the case, in which the system is locally occupied by phase 1 or phase 2 and the upper bound of $\rho$ models the saturation of surfactants at the interface.

The nonlinear coupling term $\alpha \rho \phi^{2} / 2$ ensures the same solubility of surfactants in the bulk phases, and numerically stabilizes the phase-field model [3]. The last term $-\theta \rho|\nabla \phi|^{2}$ accounts for the high concentration of surfactants near the interface $[2,36]$.

Chemical potentials are defined as the functional derivatives of the free energy $E_{\text {tot }}$ with respect to $\phi$ and $\rho$. Using the variational approach, a nonlinear Cahn-Hilliard type phase-field system for $(\phi, \rho)$ can be obtained [3, 35],

$$
\phi_{t}=M_{1} \Delta w_{\phi}
$$




$$
\begin{gathered}
w_{\phi}=-\lambda \varepsilon \Delta \phi+\lambda F^{\prime}(\phi)+\alpha \rho \phi+2 \theta \nabla \cdot(\rho \nabla \phi), \\
\rho_{t}=M_{2} \Delta w_{\rho} \\
w_{\rho}=\beta \ln \left(\frac{\rho}{1-\rho}\right)+\frac{\alpha}{2} \phi^{2}-\theta|\nabla \phi|^{2} .
\end{gathered}
$$

with periodic boundary conditions or

$$
\left.\partial_{\mathbf{n}} \phi\right|_{\Gamma}=\left.\partial_{\mathbf{n}} \rho\right|_{\Gamma}=\left.\nabla w_{\phi} \cdot \mathbf{n}\right|_{\Gamma}=\left.\nabla w_{\rho} \cdot \mathbf{n}\right|_{\Gamma}=0 .
$$

In [22], a degenerate mobility is combined with the logarithmic Flory-Huggins potential to recover the diffusion equation for $\rho$ at the extreme points $\{0,1\}$. Equation $(2.5 \mathrm{c})$ can be modified into

$$
\rho_{t}=M_{2} \nabla\left(\cdot m_{\rho} \nabla w_{\rho}\right), \quad m_{\rho}=\rho(1-\rho) .
$$

Then the PDE energy law for the governing system (2.5) can be derived directly. By taking the sum of $L^{2}$ inner product of (2.5a) with $w_{\phi},(2.5 \mathrm{~b})$ with $-\phi_{t},(2.5 \mathrm{c})$ with $w_{\rho}$, and (2.5d) with $-\rho_{t}$, we can find that the governing system satisfies the following energy law:

$$
\frac{d}{d t} E_{t o t}(\phi, \rho)=-M_{1} \int\left|\nabla w_{\phi}\right|^{2} d \Omega-M_{2} \int\left|\nabla w_{\rho}\right|^{2} d \Omega .
$$

Now we need to develop some efficient numerical schemes to solve the nonlinearly coupled BFS-PF model (2.5). The desired schemes should be accurate, easy-to-implement and energy stable. In this study, a novel SAV approach is used to design the desired numerical schemes. Following the work in [45], we regularize the Flory-Huggins potential $G(\rho)$ from domain $(0,1)$ to $(-\infty,+\infty)$. For any $\xi>0$, the regularized Flory-Huggins potential can be written as

$$
\hat{G}(\rho)=\left\{\begin{array}{lc}
\rho \ln \rho+\frac{(1-\rho)^{2}}{2 \xi}+(1-\rho) \ln \xi-\frac{\xi}{2}, & \text { if } \quad \rho \geq 1-\xi, \\
\rho \ln \rho+(1-\rho) \ln (1-\rho), & \text { if } \quad \xi \leq \rho \leq 1-\xi, \\
(1-\rho) \ln (1-\rho)+\frac{\rho^{2}}{2 \xi}+\rho \ln \xi-\frac{\xi}{2}, & \text { if } \quad \rho \leq \xi .
\end{array}\right.
$$

When $\xi \rightarrow 0, \quad \hat{G}(\rho) \rightarrow G(\rho)$, we consider the numerical solution to the model formulated with the regularized function $\hat{G}(\rho)$. We omit the ${ }^{\wedge}$ in the notation for convenience.

To ensure that the potential $G(\rho)$ is bounded form below and positive in the whole domain, we add a zero term $\beta B$ - $\beta B$ to the energy density, and the total free energy functional can be rewritten as

$$
E_{\text {tot }}(\phi, \rho)=\int\left(\frac{\lambda \varepsilon}{2}|\nabla \phi|^{2}+\frac{\lambda}{4 \varepsilon}\left(\phi^{2}-1\right)^{2}+\beta(G(\rho)+B)+\frac{\lambda \alpha}{2} \rho \phi^{2}-\theta \rho|\nabla \phi|^{2}\right) d \Omega-\beta B|\Omega| .
$$

The introduction of a positive constant $B$ ensures $G(\rho)+B>0 . B=1$ is adopted in this study. Note that the free energy is not changed due to the introduction of the zero term. Then we define two scalar auxiliary variables:

$$
U=\sqrt{E_{u}(\phi)}, \quad V=\sqrt{E_{v}(\rho)},
$$

where 


$$
E_{u}(\phi)=\int \frac{1}{4 \varepsilon}\left(\phi^{2}-1\right)^{2} d \Omega, \quad E_{v}(\rho)=\int(G(\rho)+B) d \Omega .
$$

In turn, the total free energy can be transformed into

$$
E_{t o t}(\phi, \rho, U, V)=\int\left(\frac{\lambda \varepsilon}{2}|\nabla \phi|^{2}+\frac{\alpha}{2} \rho \phi^{2}-\theta \rho|\nabla \phi|^{2}\right) d \Omega+\lambda U^{2}+\beta V^{2}-\beta B|\Omega|,
$$

and an equivalent governing system can be obtained by using the variational approach

$$
\begin{gathered}
\phi_{t}=M_{1} \Delta w_{\phi}, \\
w_{\phi}=-\lambda \varepsilon \Delta \phi+\frac{\lambda U}{\sqrt{E_{u}(\phi)}} F^{\prime}(\phi)+\alpha \rho \phi+2 \theta \nabla \cdot(\rho \nabla \phi), \\
U_{t}=\frac{1}{2 \sqrt{E_{u}(\phi)}} \int F^{\prime}(\phi) \phi_{t} d \Omega, \\
\rho_{t}=M_{2} \Delta w_{\rho} \quad \text { or } \rho_{t}=M_{2} \nabla\left(\cdot m_{\rho} \nabla w_{\rho}\right), \\
w_{\rho}=\frac{\beta V}{\sqrt{E_{v}(\rho)}} G^{\prime}(\rho)+\frac{\alpha}{2} \phi^{2}-\theta|\nabla \phi|^{2}, \\
V_{t}=\frac{1}{2 \sqrt{E_{v}(\rho)}} \int G^{\prime}(\rho) \rho_{t} d \Omega,
\end{gathered}
$$

with boundary conditions are

$$
\left.\partial_{\mathbf{n}} \phi\right|_{\Gamma}=\left.\partial_{\mathbf{n}} \rho\right|_{\Gamma}=\left.\nabla w_{\phi} \cdot \mathbf{n}\right|_{\Gamma}=\left.\nabla w_{\rho} \cdot \mathbf{n}\right|_{\Gamma}=0 .
$$

Theorem 2.1. The transformed governing system (2.11) satisfies the following energy dissipation law:

$$
\begin{gathered}
\frac{d}{d t} E_{\text {tot }}(\phi, \rho, U, V)=\frac{d}{d t} \int\left(\frac{\lambda \varepsilon}{2}|\nabla \phi|^{2}+\frac{\alpha}{2} \rho \phi^{2}-\theta \rho|\nabla \phi|^{2}\right) d \Omega+\frac{d}{d t}\left(\lambda U^{2}+\beta V^{2}\right) \\
=-M_{1} \int\left|\nabla w_{\phi}\right|^{2} d \Omega-M_{2} \int\left|\sqrt{m_{\rho}} \nabla w_{\rho}\right|^{2} d \Omega \leq 0 .
\end{gathered}
$$

Proof. By taking the inner product of equation (2.11a) with $w_{\phi}$, we have,

$$
\left(\phi_{t}, w_{\phi}\right)=\left(M_{1} \Delta w_{\phi}, w_{\phi}\right)=-M_{1} \int\left|\nabla w_{\phi}\right|^{2} d \Omega,
$$

where $(\cdot, \cdot)$ is the inner product in $L^{2}(\Omega)$.

By taking the inner product of $\alpha \rho \phi$ with $-\phi_{t}$, we obtain,

$$
\begin{array}{r}
-\left(\alpha \rho \phi, \phi_{t}\right)=-\alpha \frac{d}{d t} \int \rho \phi^{2} d \Omega+\alpha\left(\frac{d}{d t}(\rho \phi), \phi\right) \\
=-\alpha \frac{d}{d t} \int \rho \phi^{2} d \Omega+\alpha\left(\rho \phi, \phi_{t}\right)+\alpha\left(\phi^{2}, \rho_{t}\right),
\end{array}
$$

Then we can derive the following identity,

$$
-\left(\alpha \rho \phi, \phi_{t}\right)=-\frac{\alpha}{2} \frac{d}{d t} \int \rho \phi^{2} d \Omega+\frac{\alpha}{2}\left(\phi^{2}, \rho_{t}\right),
$$

By taking the inner product of $\theta \nabla \cdot(\rho \nabla \phi)$ with $-\phi_{t}$, we get 


$$
\begin{gathered}
-\left(\theta \nabla \cdot(\rho \nabla \phi), \phi_{t}\right)=\theta\left(\rho \nabla \phi, \frac{d}{d t}(\nabla \phi)\right)=\theta \frac{d}{d t} \int \rho|\nabla \phi|^{2} d \Omega-\theta\left(\frac{d}{d t}(\rho \nabla \phi), \nabla \phi\right) \\
=\theta \frac{d}{d t} \int \rho|\nabla \phi|^{2} d \Omega-\theta\left(|\nabla \phi|^{2}, \rho_{t}\right)-\theta\left(\rho \nabla \phi, \frac{d}{d t}(\nabla \phi)\right),
\end{gathered}
$$

Then we have,

$$
-2\left(\theta \nabla \cdot(\rho \nabla \phi), \phi_{t}\right)=\theta \frac{d}{d t} \int \rho|\nabla \phi|^{2} d \Omega-\theta\left(|\nabla \phi|^{2}, \rho_{t}\right),
$$

By taking the inner product of equation with $-\phi_{t}$, and using the identities (2.15) and (2.17), we can derive that

$$
\begin{gathered}
-\left(w_{\phi}, \phi_{t}\right)=\left(\lambda \varepsilon \Delta \phi, \phi_{t}\right)-\left(\lambda U b, \phi_{t}\right)-\left(\alpha \rho \phi, \phi_{t}\right)-2\left(\theta \nabla \cdot(\rho \nabla \phi), \phi_{t}\right) \\
=-\frac{\lambda \varepsilon}{2} \frac{d}{d t} \int|\nabla \phi|^{2} d \Omega-\left(\lambda U b, \phi_{t}\right)-\frac{\alpha}{2} \frac{d}{d t} \int \rho \phi^{2} d \Omega+\frac{\alpha}{2}\left(\phi^{2}, \rho_{t}\right) \\
+\theta \frac{d}{d t} \int \rho|\nabla \phi|^{2} d \Omega-\theta\left(|\nabla \phi|^{2}, \rho_{t}\right),
\end{gathered}
$$

where

$$
b=\frac{F^{\prime}(\phi)}{\sqrt{E_{u}(\phi)}},
$$

By taking the inner product of equation (2.11c) with $2 \lambda U$, we get

$$
\left(U_{t}, 2 \lambda U\right)=\lambda \frac{d}{d t} U^{2}=\left(\lambda U b, \phi_{t}\right),
$$

Summing up equations (2.13), (2.18) and (2.19), we obtain

$$
\begin{aligned}
& \lambda \frac{d}{d t} U^{2}=-\frac{\lambda \varepsilon}{2} \frac{d}{d t} \int|\nabla \phi|^{2} d \Omega-\frac{\alpha}{2} \frac{d}{d t} \int \rho \phi^{2} d \Omega+\frac{\alpha}{2}\left(\phi^{2}, \rho_{t}\right) \\
& \quad+\theta \frac{d}{d t} \int \rho|\nabla \phi|^{2} d \Omega-\theta\left(|\nabla \phi|^{2}, \rho_{t}\right),
\end{aligned}
$$

By taking the inner product of equation (2.11d) with $w_{\rho}$, we get

$$
\left(\rho_{t}, w_{\rho}\right)=\left(M_{2} \Delta w_{\rho}, w_{\rho}\right)=-M_{2} \int\left|\sqrt{m_{\rho}} \nabla w_{\rho}\right|^{2} d \Omega,
$$

By taking the inner product of equation (2.11e) with $-\rho_{t}$, we get

$$
-\left(w_{\rho}, \rho_{t}\right)=-\left(\beta V a, \rho_{t}\right)-\frac{\alpha}{2}\left(\phi^{2}, \rho_{t}\right)+\theta\left(|\nabla \phi|^{2}, \rho_{t}\right),
$$

where

$$
a=\frac{G^{\prime}(\rho)}{\sqrt{E_{v}(\rho)}},
$$

By taking the inner product of equation (2.11f) with $2 \beta V$, we have

$$
\left(V_{t}, 2 \beta V\right)=\beta \frac{d}{d t} V^{2}=\left(V a, \rho_{t}\right),
$$

Summing up equations (2.20) - (2.23), we get the desired results (2.12)

It is clear that the transformed system (2.11) satisfies the exactly same energy dissipation law with the original system (2.6) for the time-continuous case. In the next section, we will focus on constructing time-marching schemes for the transformed governing system.

\section{Linear, decoupled and energy stable schemes}




\subsection{First-order scheme}

We now present two different first-order schemes to solve the governing system (2.11).

Scheme 1. Given $\rho^{n}, \phi^{n}, V^{n}$ and $U^{n}$, the scheme calculates $\rho^{n+1}, \phi^{n+1}, V^{n+1}$ and $U^{n+1}$ for $n \geq 0$ in two steps.

Step 1, we update $\rho^{n+1}$ and $V^{n+1}$ by solving

$$
\begin{gathered}
\frac{\rho^{n+1}-\rho^{n}}{\delta t}-M_{2} \Delta w_{\rho}^{n+1}=0 \quad \text { or } \quad \frac{\rho^{n+1}-\rho^{n}}{\delta t}-M_{2} \nabla \cdot\left(m_{\rho}^{n} \nabla w_{\rho}^{n+1}\right)=0, \\
w_{\rho}^{n+1}=\frac{\beta V^{n+1}}{\sqrt{E_{v}\left(\rho^{n}\right)}} G^{\prime}\left(\rho^{n}\right)+\frac{\alpha}{2}\left(\phi^{n}\right)^{2}-\theta\left|\nabla \phi^{n}\right|^{2}, \\
\frac{V^{n+1}-V^{n}}{\delta t}=\frac{1}{2 \sqrt{E_{v}\left(\rho^{n}\right)}} \int_{\Omega} G^{\prime}\left(\rho^{n}\right) \frac{\left(\rho^{n+1}-\rho^{n}\right)}{\delta t} d \Omega, \\
\left.\partial_{\mathbf{n}} \rho^{n+1}\right|_{\Gamma}=0,\left.\partial_{\mathbf{n}} w_{\rho}^{n+1}\right|_{\Gamma}=0,
\end{gathered}
$$

Step 2, updating $\phi^{n+1}$ and $U^{n+1}$ using

$$
\begin{gathered}
\frac{\phi^{n+1}-\phi^{n}}{\delta t}-M_{1} \Delta w_{\phi}^{n+1}=0, \\
w_{\phi}^{n+1}=-\lambda \varepsilon \Delta \phi^{n+1}+\frac{\lambda U^{n+1}}{\sqrt{E_{u}\left(\phi^{n}\right)}} F^{\prime}\left(\phi^{n}\right)+\alpha \rho^{n+1} \phi^{n+1}+\theta \nabla \cdot\left(\rho^{n+1} \nabla\left(\phi^{n+1}+\phi^{n}\right)\right), \\
\frac{U^{n+1}-U^{n}}{\delta t}=\frac{1}{2 \sqrt{E_{u}\left(\phi^{n}\right)}} \int_{\Omega} F^{\prime}\left(\phi^{n}\right) \frac{\left(\phi^{n+1}-\phi^{n}\right)}{\delta t} d \Omega, \\
\left.\partial_{\mathbf{n}} \phi^{n+1}\right|_{\Gamma}=0,\left.\partial_{\mathbf{n}} w_{\phi}^{n+1}\right|_{\Gamma}=0 .
\end{gathered}
$$

Now we take the computation of $\rho^{n+1}$ as an example to give readers a clear understanding of the semi-implicit scheme (3.1). Equation (3.1c) can be rewritten as

$$
V^{n+1}=V^{n}-\frac{1}{2}\left(a^{n}, \rho^{n}\right)+\frac{1}{2}\left(a^{n}, \rho^{n+1}\right),
$$

where

$$
a^{n}=\frac{G^{\prime}\left(\rho^{n}\right)}{\sqrt{E_{v}\left(\rho^{n}\right)}}
$$

Substituting (3.2) into (3.1b), we can easily derive that

$$
\begin{aligned}
w_{\rho}^{n+1} & =\beta a^{n}\left[V^{n}-\frac{1}{2}\left(a^{n}, \rho^{n}\right)+\frac{1}{2}\left(a^{n}, \rho^{n+1}\right)\right]+\frac{\alpha}{2}\left(\phi^{n}\right)^{2}-\theta\left|\nabla \phi^{n}\right|^{2} \\
& =\frac{\beta}{2}\left(a^{n}, \rho^{n+1}\right) a^{n}+\beta\left[V^{n}-\frac{1}{2}\left(a^{n}, \rho^{n}\right)\right] a^{n}+\frac{\alpha}{2}\left(\phi^{n}\right)^{2}-\theta\left|\nabla \phi^{n}\right|^{2} \\
& =\frac{\beta}{2}\left(a^{n}, \rho^{n+1}\right) a^{n}+g^{n}
\end{aligned}
$$

where

$$
g^{n}=\beta\left[V^{n}-\frac{1}{2}\left(a^{n}, \rho^{n}\right)\right] a^{n}+\frac{\alpha}{2}\left(\phi^{n}\right)^{2}-\theta\left|\nabla \phi^{n}\right|^{2}
$$


If denoting $A=-\Delta$, and substituting (3.3) into (3.1a), we can easily derive that

$$
\rho^{n+1}+\frac{\delta t M_{2} \beta}{2}\left(a^{n}, \rho^{n+1}\right) A a^{n}=s^{n}
$$

where

$$
s^{n}=\rho^{n}-\delta t M_{2} A g^{n}
$$

By taking the inner product of equation (3.4) with $a^{n}$, we have

$$
\left(a^{n}, \rho^{n+1}\right)+\frac{\delta t M_{2} \beta}{2}\left(a^{n}, \rho^{n+1}\right)\left(a^{n}, A a^{n}\right)=s^{n}
$$

then we get

$$
\left(a^{n}, \rho^{n+1}\right)=\frac{2 s^{n}}{\delta t M_{2} \beta\left(a^{n}, A a^{n}\right)+2} .
$$

With $\phi^{n}, \rho^{n}$ and $V^{n}, \rho^{n+1}$ can be obtained from the equation (3.4) in a "explicit" way. The computations of $\rho^{n+1}$ and $\phi^{n+1}$ are totally decoupled. Once $\rho^{n+1}$ is obtained from step 1, we can calculate $\phi^{n+1}$ follow the procedure (3.2) -(3.6). To simplify the presentation, we omit the computational details of $\phi^{n+1}$. At each time step, the computation of $\phi^{n+1}$ involves solving two linear elliptic equations. The introduction of $U$ and $V$ allow the nonlinear potentials to be treated semi-implicitly. With $\rho^{n+1}$ and $\phi^{n+1}, V^{n+1}$ and $U^{n+1}$ are automatically obtained from equations (3.1c) and $(3.1 \mathrm{~g})$, and thus do not involve extra computational costs. Form the above analysis we can see that the constructed scheme is very efficient and easy-to-implement. Next, we carry out the energy estimates for the scheme (3.1).

Theorem 3.1. The linear, decoupled scheme (3.1) is unconditionally energy stable, and satisfies the following discrete energy law:

$$
E^{n+1}-E^{n}=-\delta t M_{1}\left\|\nabla w_{\phi}^{n+1}\right\|^{2}-\delta t M_{2}\left\|\sqrt{m_{\rho}^{n}} \nabla w_{\rho}^{n+1}\right\|^{2} \leq 0,
$$

where

$$
E^{n}=\frac{\lambda \varepsilon}{2}\left\|\nabla \phi^{n}\right\|^{2}+\lambda\left\|U^{n}\right\|^{2}+\beta\left\|V^{n}\right\|^{2}+\frac{\alpha}{2}\left(\rho^{n},\left|\phi^{n}\right|^{2}\right)-\theta\left(\rho^{n},\left|\nabla \phi^{n}\right|^{2}\right) .
$$

here $\|\cdot\|$ denotes the $L^{2}$-norm in $\Omega$.

Proof. By taking the inner product of (3.1a) with $\delta t w_{\rho}^{n+1}$, we can easily derive that

$$
\left(\rho^{n+1}-\rho^{n}, w_{\rho}^{n+1}\right)=-\delta t M_{2}\left\|\sqrt{m_{\rho}^{n}} \nabla w_{\rho}^{n+1}\right\|^{2},
$$

By taking the inner product of (3.1b) with $-\left(\rho^{n+1}-\rho^{n}\right)$ and applying the following identity

$$
(a-b, 2 a)=|a|^{2}-|b|^{2}+|a-b|^{2},
$$

we obtain,

$$
\begin{aligned}
& -\left(\rho^{n+1}-\rho^{n}, w_{\rho}^{n+1}\right)=-\left(\beta V^{n+1} a^{n}, \rho^{n+1}-\rho^{n}\right)-\frac{\alpha}{2}\left(\rho^{n+1}-\rho^{n},\left|\phi^{n}\right|^{2}\right) \\
& \quad+\theta\left(\rho^{n+1}-\rho^{n},\left|\nabla \phi^{n}\right|^{2}\right),
\end{aligned}
$$

By taking the inner product of (3.1c) with $2 \beta V^{n+1}$ we obtain 


$$
\beta\left(\left\|V^{n+1}\right\|^{2}-\left\|V^{n}\right\|^{2}+\left\|V^{n+1}-V^{n}\right\|^{2}\right)=\left(\beta V^{n+1} a^{n}, \rho^{n+1}-\rho^{n}\right),
$$

Summing up equations (3.4) - (3.6), we get

$$
\begin{aligned}
& \beta\left(\left\|V^{n+1}\right\|^{2}-\left\|V^{n}\right\|^{2}+\left\|V^{n+1}-V^{n}\right\|^{2}\right)=-\delta t M_{2}\left\|\sqrt{m_{\rho}^{n}} \nabla w_{\rho}^{n+1}\right\|^{2}-\frac{\alpha}{2}\left(\rho^{n+1}-\rho^{n},\left|\phi^{n}\right|^{2}\right) \\
& +\theta\left(\rho^{n+1}-\rho^{n},\left|\nabla \phi^{n}\right|^{2}\right),
\end{aligned}
$$

By taking the inner product of (3.1e) with $\delta t w_{\phi}^{n+1}$, we have

$$
\left(\phi^{n+1}-\phi^{n}, w_{\phi}^{n+1}\right)=-\delta t M_{1}\left\|\nabla w_{\phi}^{n+1}\right\|^{2},
$$

By taking the $L^{2}$ inner product of (3.1f) with $-\left(\phi^{n+1}-\phi^{n}\right)$, we obtain

$$
\begin{gathered}
-\left(\phi^{n+1}-\phi^{n}, w_{\phi}^{n+1}\right)=-\frac{\lambda \varepsilon}{2}\left(\left\|\nabla \phi^{n+1}\right\|^{2}-\left\|\nabla \phi^{n}\right\|^{2}+\left\|\nabla \phi^{n+1}-\nabla \phi^{n}\right\|^{2}\right) \\
-\left(\lambda U^{n+1} b^{n}, \phi^{n+1}-\phi^{n}\right)+\theta\left(\rho^{n+1},\left|\nabla \phi^{n+1}\right|^{2}-\left|\nabla \phi^{n}\right|^{2}\right) \\
-\frac{\alpha}{2}\left[\left(\rho^{n+1},\left|\phi^{n+1}\right|^{2}\right)-\left(\rho^{n+1},\left|\phi^{n}\right|^{2}\right)+\left(\rho^{n+1},\left|\phi^{n+1}-\phi^{n}\right|^{2}\right)\right],
\end{gathered}
$$

where

$$
b^{n}=\frac{F^{\prime}\left(\phi^{n}\right)}{\sqrt{E_{u}\left(\phi^{n}\right)}},
$$

By taking the inner product of $(3.1 \mathrm{~g})$ with $2 \lambda U^{n+1}$, we get

$$
\lambda\left(\left\|U^{n+1}\right\|^{2}-\left\|U^{n}\right\|^{2}+\left\|U^{n+1}-U^{n}\right\|^{2}\right)=\left(\lambda U^{n+1} b^{n}, \phi^{n+1}-\phi^{n}\right),
$$

Summing up all equations (3.7) - (3.10), we have

$$
\begin{aligned}
& \frac{\lambda \varepsilon}{2}\left(\left\|\nabla \phi^{n+1}\right\|^{2}-\left\|\nabla \phi^{n}\right\|^{2}+\left\|\nabla \phi^{n+1}-\nabla \phi^{n}\right\|^{2}\right)+\lambda\left(\left\|U^{n+1}\right\|^{2}-\left\|U^{n}\right\|^{2}+\left\|U^{n+1}-U^{n}\right\|^{2}\right) \\
& \quad+\beta\left(\left\|V^{n+1}\right\|^{2}-\left\|V^{n}\right\|^{2}+\left\|V^{n+1}-V^{n}\right\|^{2}\right)-\theta\left[\left(\rho^{n+1},\left|\nabla \phi^{n+1}\right|^{2}\right)-\left(\rho^{n},\left|\nabla \phi^{n}\right|^{2}\right)\right] \\
& \quad+\frac{\alpha}{2}\left[\left(\rho^{n+1},\left|\phi^{n+1}\right|^{2}\right)-\left(\rho^{n},\left|\phi^{n}\right|^{2}\right)+\left(\rho^{n+1},\left|\phi^{n+1}-\phi^{n}\right|^{2}\right)\right] \\
& \quad=-M_{1}\left\|\nabla w_{\phi}^{n+1}\right\|^{2}-M_{2}\left\|\sqrt{m_{\rho}^{n}} \nabla w_{\rho}^{n+1}\right\|^{2} \leq 0 .
\end{aligned}
$$

which implies the desired results

Remark 3.1. By using the SAV approach, we develop the first linear, decoupled and unconditionally energy stable scheme (3.1) for the BFS-PF model. Compared with the traditional time stepping schemes, e.g., the IEQ approach, the SAV approach only requires the nonlinear parts of free energy are bounded form below, which is necessary for the free energy to be physically sound. Also, this approach is not restricted to the specific forms of nonlinear parts of free energy, and it works well for the fourth-order double well potential and logarithmic Flory-Huggins potential in this study. With the introduction of two scalar variables $U$ and $V$, the nonlinear potentials are transformed into equivalent forms, providing the fundamental support for semi-explicit processing. Furthermore, the proposed schemes in this study can be extended to a 
large class of gradient flow problems with multiple variables.

The scheme (3.1) calculates $\rho^{n+1}$ first, and then calculates $\phi^{n+1}$. Now we present the other first-order scheme, in which $\phi^{n+1}$ is computed first.

Scheme 2. Given $\phi^{n}, \rho^{n}, U^{n}$ and $V^{n}$, the scheme calculates $\phi^{n+1}, \rho^{n+1}, U^{n+1}$ and $V^{n+1}$ for $n \geq 0$ in two steps.

Step 1, we update $\phi^{n+1}$ and $U^{n+1}$ by solving

$$
\begin{gathered}
\frac{\phi^{n+1}-\phi^{n}}{\delta t}-M_{1} \Delta w_{\phi}^{n+1}=0, \\
w_{\phi}^{n+1}=-\lambda \varepsilon \Delta \phi^{n+1}+\frac{\lambda U^{n+1}}{\sqrt{E_{u}\left(\phi^{n}\right)}} F^{\prime}\left(\phi^{n}\right)+\alpha \rho^{n} \phi^{n+1}+\theta \nabla \cdot\left(\rho^{n} \nabla\left(\phi^{n+1}+\phi^{n}\right)\right), \\
\frac{U^{n+1}-U^{n}}{\delta t}=\frac{1}{2 \sqrt{E_{u}\left(\phi^{n}\right)}} \int_{\Omega} F^{\prime}\left(\phi^{n}\right) \frac{\left(\phi^{n+1}-\phi^{n}\right)}{\delta t} d \Omega, \\
\left.\partial_{\mathbf{n}} \phi^{n+1}\right|_{\Gamma}=0,\left.\partial_{\mathbf{n}} w_{\phi}^{n+1}\right|_{\Gamma}=0,
\end{gathered}
$$

Step 2, updating $\rho^{n+1}$ and $V^{n+1}$ using

$$
\begin{gathered}
\frac{\rho^{n+1}-\rho^{n}}{\delta t}-M_{2} \Delta w_{\rho}^{n+1}=0 \quad \text { or } \quad \frac{\rho^{n+1}-\rho^{n}}{\delta t}-M_{2} \nabla \cdot\left(m_{\rho}^{n} \nabla w_{\rho}^{n+1}\right)=0, \\
w_{\rho}^{n+1}=\frac{\beta V^{n+1}}{\sqrt{E_{v}\left(\rho^{n}\right)}} G^{\prime}\left(\rho^{n}\right)+\frac{\alpha}{2}\left(\phi^{n+1}\right)^{2}-\theta\left|\nabla \phi^{n+1}\right|^{2}, \\
\frac{V^{n+1}-V^{n}}{\delta t}=\frac{1}{2 \sqrt{E_{v}\left(\rho^{n}\right)}} \int_{\Omega} G^{\prime}\left(\rho^{n}\right) \frac{\left(\rho^{n+1}-\rho^{n}\right)}{\delta t} d \Omega, \\
\left.\partial_{\mathbf{n}} \rho^{n+1}\right|_{\Gamma}=0,\left.\partial_{\mathbf{n}} w_{\rho}^{n+1}\right|_{\Gamma}=0 .
\end{gathered}
$$

In the scheme (3.12), the computations of $\rho^{n+1}$ and $\phi^{n+1}$ are totally decoupled. Furthermore, $\rho^{n+1}$ can be calculated directly, without solving any partial differential equations. Once we obtained $\rho^{n+1}$ and $\phi^{n+1}, U^{n+1}$ and $V^{n+1}$ are be calculated explicitly. The above indicates that the scheme (3.12) is extremely efficient.

Theorem 3.2. The linear, decoupled scheme (3.12) is unconditionally energy, and satisfies the following discrete energy law:

$$
E^{n+1}-E^{n}=-M_{1}\left\|\nabla w_{\phi}^{n+1}\right\|^{2}-M_{2}\left\|\sqrt{m_{\rho}^{n}} \nabla w_{\rho}^{n+1}\right\|^{2} \leq 0 .
$$

Proof. We omit the detailed derivations here since the proof is similar to that for Theorem 3.1.

\subsection{Second-order scheme}

In terms of stability, the unconditionally energy stable schemes have no constraints on time step size. However, a large time step size will definitely introduce large errors in practice, which motivates us to construct high-order schemes, e.g., second-order schemes. We extend the above first-order schemes to a second-order version based on the BDF2. Given $\rho^{n-1}, \phi^{n-1}, U^{n-1}, V^{n-1}$, and $\rho^{n}, \phi^{n}, V^{n}, U^{n}$, the scheme calculates $\rho^{n+1}, \phi^{n+1}, U^{n+1}$ and $V^{n+1}$ in two steps.

Step 1, we update $\phi^{n+1}$ and $U^{n+1}$ by solving 


$$
\left\{\begin{array}{l}
\frac{3 \phi^{n+1}-4 \phi^{n}+\phi^{n-1}}{2 \delta t}-M_{1} \Delta w_{\phi}^{n+1}=0 \\
w_{\phi}^{n+1}=-\lambda \varepsilon \Delta \phi^{n+1}+\frac{\lambda U^{n+1}}{\sqrt{E_{1}\left(\phi^{*}\right)}} F^{\prime}\left(\phi^{*}\right)+\alpha \rho^{*} \phi^{n+1}+\theta \nabla \cdot\left(\rho^{*} \nabla\left(\phi^{n+1}+\phi^{*}\right)\right) \\
\frac{3 U^{n+1}-4 U^{n}+U^{n-1}}{2 \delta t}=\frac{1}{2 \sqrt{E_{u}\left(\phi^{*}\right)}} \int_{\Omega} F^{\prime}\left(\phi^{*}\right) \frac{\left(3 \phi^{n+1}-4 \phi^{n}+\phi^{n-1}\right)}{2 \delta t} d \Omega \\
\left.\partial_{\mathbf{n}} \phi^{n+1}\right|_{\Gamma}=0,\left.\partial_{\mathbf{n}} w_{\phi}^{n+1}\right|_{\Gamma}=0
\end{array}\right.
$$

Step 2, Updating $\rho^{n+1}$ and $V^{n+1}$ as follows,

$$
\left\{\begin{array}{l}
\frac{3 \rho^{n+1}-4 \rho^{n}+\rho^{n-1}}{2 \delta t}-M_{2} \Delta w_{\rho}^{n+1}=0 \quad \text { or } \quad \frac{3 \rho^{n+1}-4 \rho^{n}+\rho^{n-1}}{2 \delta t}-M_{2} \nabla \cdot\left(m_{\rho}^{*} \nabla w_{\rho}^{n+1}\right)=0 \\
w_{\rho}^{n+1}=\frac{\beta V^{n+1}}{\sqrt{E_{2}\left(\rho^{*}\right)}} G^{\prime}\left(\rho^{*}\right)+\frac{\alpha}{2}\left(\phi^{n+1}\right)^{2}-\theta\left|\nabla \phi^{n+1}\right|^{2}, \\
\frac{3 V^{n+1}-4 V^{n}+V^{n-1}}{2 \delta t}=\frac{1}{2 \sqrt{E_{v}\left(\rho^{*}\right)}} \int_{\Omega} G^{\prime}\left(\rho^{*}\right) \frac{\left(3 \rho^{n+1}-4 \rho^{n}+\rho^{n-1}\right)}{2 \delta t} d \Omega, \\
\left.\partial_{\mathbf{n}} \rho^{n+1}\right|_{\Gamma}=0,\left.\partial_{\mathbf{n}} w_{\rho}^{n+1}\right|_{\Gamma}=0,
\end{array}\right.
$$

where

$$
\phi^{*}=2 \phi^{n}-\phi^{n-1}, \quad \rho^{*}=2 \rho^{n}-\rho^{n-1}, \quad m_{\rho}^{*}=2 m_{\rho}^{n}-m_{\rho}^{n-1} .
$$

The nonlinearly coupled terms between the phase variables present a huge challenge to carry out the energy estimates. Although the unconditional stability of the second-order schemes still needs to be proved, a series of numerical tests performed in section 4.1 have shown the energy stability of the proposed second-order scheme.

\section{Numerical results.}

In this section, we conduct a series of numerical experiments to validate the accuracy, efficiency and the energy decay property of the proposed numerical schemes.

\subsection{Accuracy test}

To test the accuracy of the proposed schemes, we simulate the evolution of a square shaped fluid bubble with a side length $\pi$ in the domain of $[0,2 \pi] \times[0,2 \pi]$. The square bubble is located in the center of the domain with a side length $\pi$, as shown in Figure 1. The initial concentration of surfactants in the bulk phases $\rho_{0}$ is set as 0.3 . The other parameters used in the simulations are as follows:

$$
M_{1}=0.01, \quad M_{2}=0.01, \quad \alpha=0.001, \quad \beta=0.05, \quad \varepsilon=0.05, \quad \theta=0.001, \quad B=1 .
$$

We use LS1_tho, LS1_ph and LS2 to represent the schemes (3.1), (3.12) and (3.14), respectively. A spatial resolution $n_{x}=150, n_{y}=150$ is used to reduce the error related to the spatial discretization. Because of the absence of the exact solution, the solution obtained by LS2 with the time step $\Delta t=7.8125 \times 10^{-5}$ is selected as the reference solution. Various time steps $\Delta t=5 \times 10^{-3}$, $2.5 \times 10^{-3}, 1.25 \times 10^{-3}, 6.25 \times 10^{-4}, 3.125 \times 10^{-4}$ and $1.5625 \times 10^{-4}$ are used to calculate the $L^{2}$-norm errors [46] and convergence rate [47] for the phase variable $\phi$ at $t=1$. It can be observed in Table 1 that the schemes LS1_tho and LS1_ph achieve the first-order accuracy in time, and LS2 can 
achieve the second accuracy in time. Moreover, the scheme LS1_tho gives smaller error than the scheme LS1_ph and the second-order scheme LS2 gives better accuracy than the first-order scheme at the same time step size.

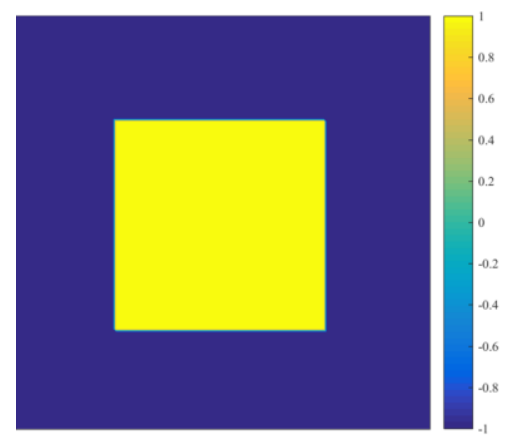

Figure 1 A square bubble (yellow phase) with a side length $\pi$ in the domain of $[0,2 \pi] \times[0,2 \pi]$.

Table 1. The $L^{2}$ norm error and convergence rate for the phase variable $\phi$ at $t=1$ with different temporal resolutions.

\begin{tabular}{|c|c|c|c|c|c|c|}
\hline$\Delta t$ & LS1_tho & Order & LS1_ph & Order & LS2 & Order \\
\hline $5 \mathrm{E}-03$ & $6.99 \mathrm{E}-03$ & - & $6.81 \mathrm{E}-03$ & - & $6.53 \mathrm{E}-04$ & - \\
\hline $2.5 \mathrm{E}-03$ & $2.99 \mathrm{E}-03$ & 1.01 & $3.38 \mathrm{E}-03$ & 1.22 & $1.88 \mathrm{E}-04$ & 1.80 \\
\hline $1.25 \mathrm{E}-03$ & $1.34 \mathrm{E}-03$ & 1.04 & $1.64 \mathrm{E}-03$ & 1.16 & $4.92 \mathrm{E}-05$ & 1.93 \\
\hline $6.25 \mathrm{E}-04$ & $6.69 \mathrm{E}-04$ & 1.10 & $7.68 \mathrm{E}-04$ & 1.00 & $1.22 \mathrm{E}-05$ & 2.01 \\
\hline $3.125 \mathrm{e}-4$ & $3.04 \mathrm{E}-04$ & 1.23 & $3.28 \mathrm{E}-04$ & 1.14 & $2.81 \mathrm{E}-06$ & 2.12 \\
\hline $1.5625 \mathrm{e}-4$ & $1.07 \mathrm{E}-04$ & 1.59 & $1.09 \mathrm{E}-04$ & 1.50 & $6.78 \mathrm{E}-07$ & 2.05 \\
\hline
\end{tabular}

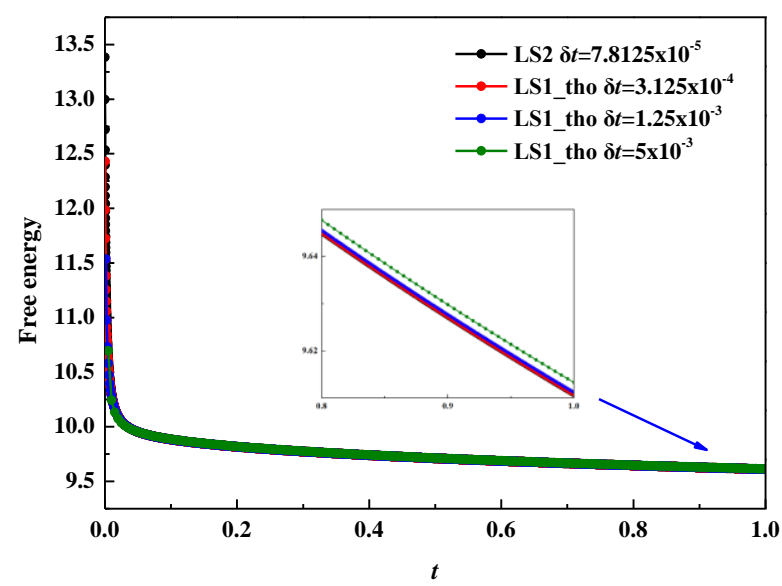

Figure 2 Time evolution of the free energy $E_{\text {tot }}$ for different time step sizes. The trend of all energy curves demonstrates that our scheme is unconditionally energy stable. $\left(n_{x}=n_{y}=150, M_{1}=M_{2}=0.01, \alpha=0.001, \beta=0.05, \varepsilon\right.$ $\left.=0.05, \theta=1 \times 10^{-3}\right)$.

The energy curves for all time step sizes in Figure 2 confirm the unconditional energy stability of the proposed scheme.

\subsection{Surfactant absorption}

The process of phase separation in a two-dimensional domain of $[0,2 \pi] \times[0,2 \pi]$ is considered in this section to demonstrate the adsorption behavior of surfactants on the phase interface. The following parameters are used:

$$
M_{1}=0.05, \quad M_{2}=0.05, \quad \alpha=0.001, \quad \beta=0.05, \quad \varepsilon=0.05, \quad \theta=8 \times 10^{-4}, \quad \lambda=1 .
$$


Initially, the phase field variable $\phi$ is set as the constant value with random perturbation in the whole domain associated, and the surfactant concentration variable $\rho$ is locally concentrated at the center of domain. The specific profiles are chosen as

$$
\begin{gathered}
\phi_{0}=0.2+0.001 \operatorname{rand}(x, y), \\
\rho_{0}=0.25-0.15 \tanh \left(\frac{1}{\sqrt{2} \varepsilon}\left(\sqrt{(x-\pi)^{2}+(y-\pi)^{2}}-1\right)\right),
\end{gathered}
$$

where the rand $(x, y)$ is the random number in $[-1,1]$ and has zero mean. We choose the spatial resolution $n_{x}=150, n_{y}=150$, time step size $\Delta t=1 \times 10^{-3}$ and use the scheme LS2 (better accuracy) to simulate the phase separation process. Figure 3 gives the dynamical behaviors of $\phi$ and $\rho$ at different times. It can be observed that the concentrated surfactants slowly absorbed onto the interface.
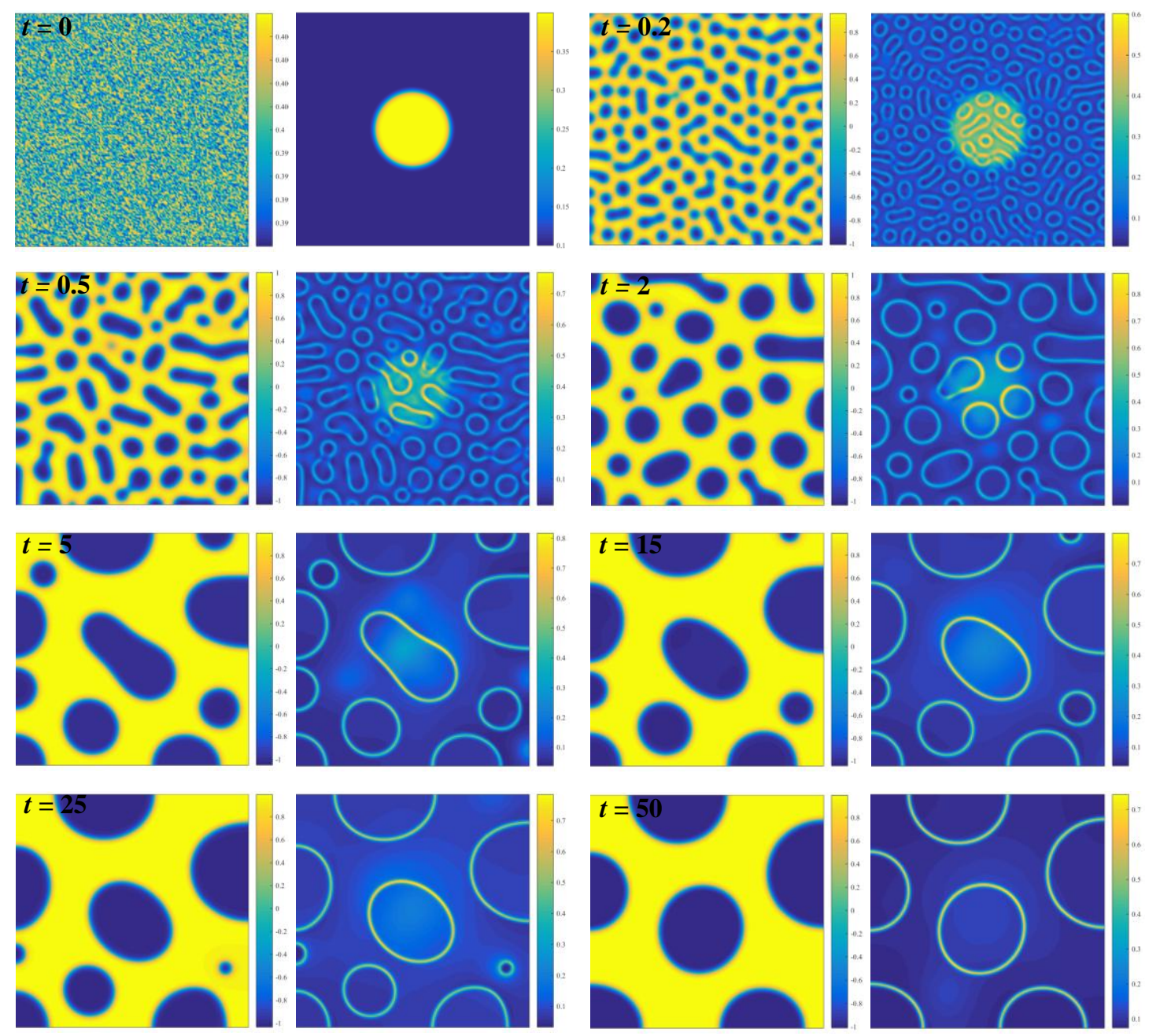

Figure 3 Evolutions of the phase variables $\phi$ and $\rho$ at different times. For each subfigure, the left is the profile of $\phi$, and the right is the profile of $\rho$. Surfactants are slowly absorbed onto the interface with the separation of two phases. Some important parameters used in this simulation: $n_{x}=n_{y}=150, \Delta t=1 \times 10^{-3}, M_{1}=M_{2}=0.05, \alpha=0.001, \beta=$ $0.05, \varepsilon=0.05, \theta=8 \times 10^{-4}$. 


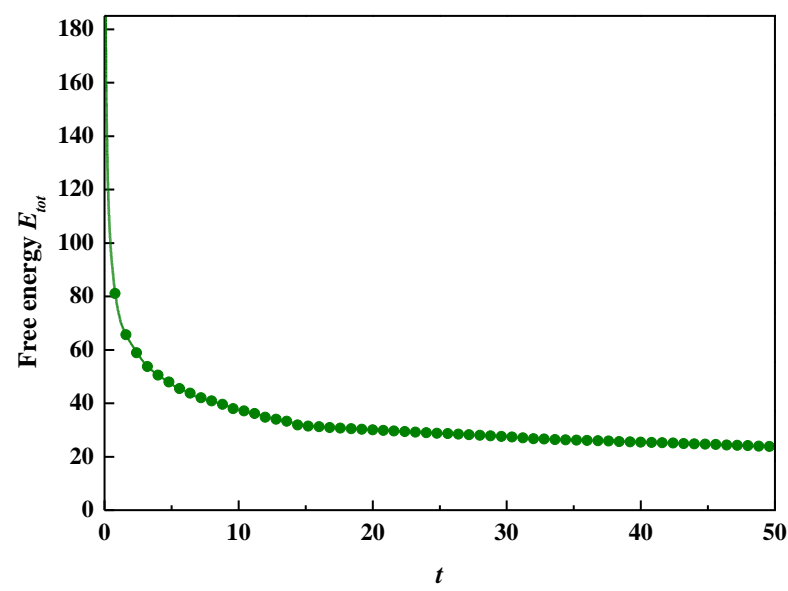

Figure 4 Time evolution of the free energy $E_{t o t}$. The energy decay curve demonstrates that our scheme is unconditionally energy stable. Some important parameters and computational details can refer to the caption of Figure 3.

Figure 4 indicates that the proposed scheme LS2 is energy stable. After the interfacial regime has emerged, the free energy $E_{t o t}$ is proportional to the interface length. Thus, the evolution of $E_{t o t}$ can be used to quantitatively characterizing the coarsening dynamics [48]. We give the evolution of $E_{\text {tot }}$ on a $\log$-log plot. In Figure 5, the free energy goes according to $E_{t o t} \approx C t^{-1 / 3}$ within the time window [3, 10], which is consistent with the results in [48].

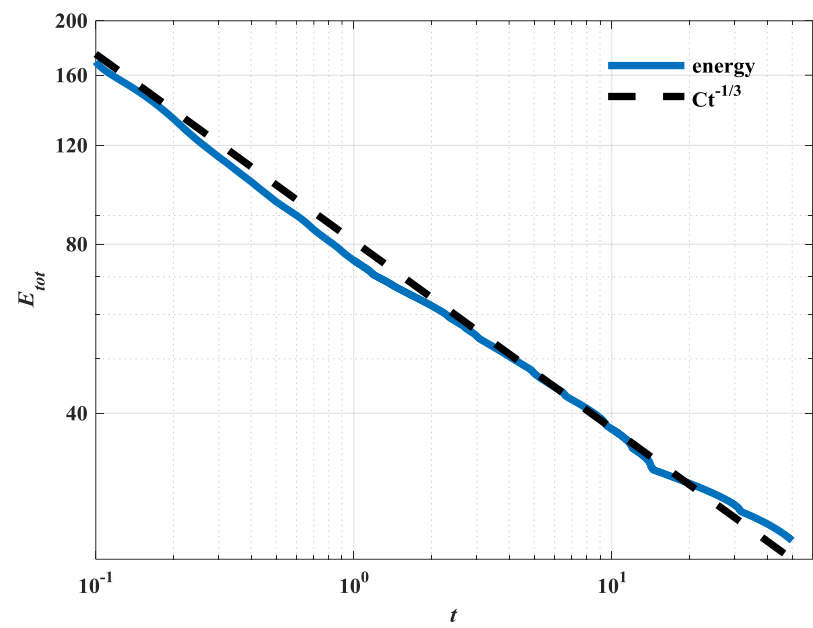

Figure 5 Time evolution of the free energy (double logarithmic scale, $t=0.1$ to 50 ). The free energy goes according to $E_{t o t} \approx C t^{-1 / 3}(C=81)$ within $t=3$ to 10 . Some important parameters and computational details can refer to the caption of Figure 3.

\subsection{Coarsening dynamics}

We first study the two-dimensional coarsening dynamics with a degenerate mobility $m_{\rho}$. The computational domain is $[0,2 \pi] \times[0,2 \pi]$. We use a grid size of $150^{2}$ and time step size $\delta t=1 \times 10^{-3}$. Other simulation parameters include:

$$
M_{1}=1 \times 10^{-3}, \quad M_{2}=0.01, \quad \alpha=1 \times 10^{-3}, \quad \beta=0.05, \quad \varepsilon=0.05, \quad \theta=1 \times 10^{-4}, \quad \lambda=1 .
$$

The initial conditions are taken as the randomly perturbed concentration fields as follows:

$$
\phi_{0}=0.001 \operatorname{rand}(x, y) \text {, }
$$




$$
\rho_{0}=0.01+0.01 \operatorname{rand}(x, y),
$$

To demonstrate the effect of a degenerate mobility, a case with the constant mobility $M_{2}=$ 0.01 is performed as a reference. Compared with the case with constant mobility, the introduction of $m_{\rho}$ decreases the migration speed of surfactants, resulting in the higher surfactant bulk concentration in many places of domain, as shown in Figure 6. We note that a nonlinear mobility also leads to the non-uniform surfactant distribution along the interface. The quantitative comparison of surfactant concentrations in Figure 7 further verifies this conclusion. Similar to Figure 5, the free energy goes according to $E_{t o t} \approx C t^{-1 / 3}(C=271)$ within the time window $[15,50]$ in Figure 8.
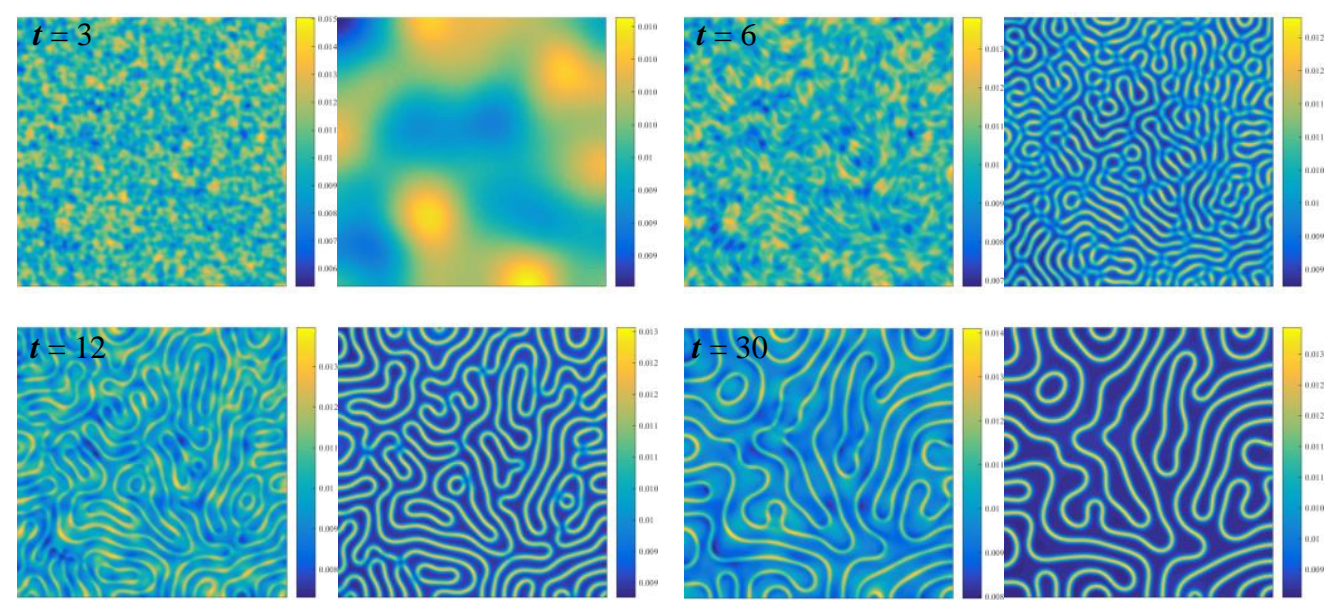

Figure 6 Evolutions of the phase variable $\rho$ at different times. For each subfigure, the left is the case with a nonlinear mobility $\left(M_{2}=0.01, m_{\rho}=\rho \times(1-\rho)\right)$, and the right is the case with a constant mobility $\left(M_{2}=1 \times 10^{-3}\right)$. Some important parameters used in this simulation: $n_{x}=n_{y}=150, \Delta t=1 \times 10^{-3}, M_{1}=1 \times 10^{-3}, \alpha=1 \times 10^{-3}, \beta=0.05, \varepsilon=$ $0.05, \theta=1 \times 10^{-4}$.

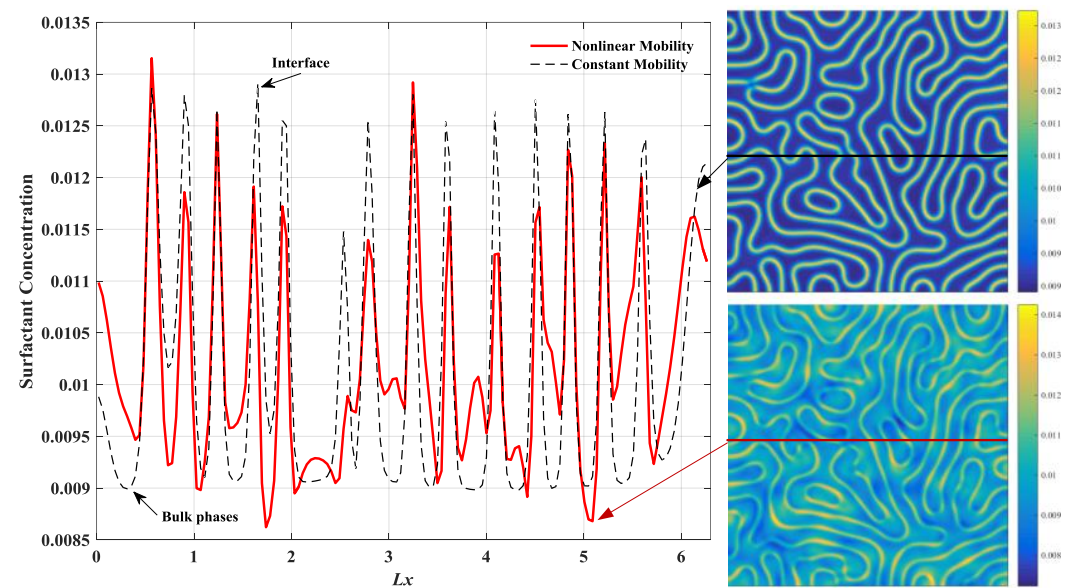

Figure 7 Cross-sectional concentration of surfactants (along $y=\pi$ ) at $t=18$. Red solid line: nonlinear mobility; black dash line: constant mobility. Some important parameters and computational details can refer to the caption of Figure 6. 


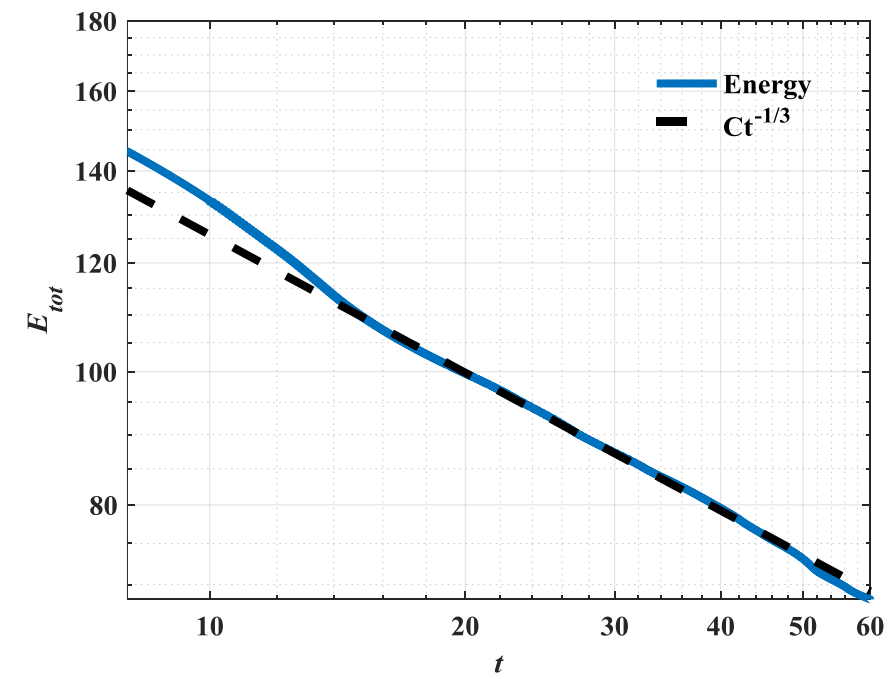

Figure 8 Time evolution of free energy (double logarithmic scale, $t=10$ to 60). Free energy goes according to $E_{t o t}$ $\approx C t^{-1 / 3}(C=271)$ within $t=15$ to 50 . Some important parameters and computational details can refer to the caption of Figure 6 .

We continue to extend the coarsening dynamics (constant mobility) to a 3D domain of $[0,1]$ $\times[0,1] \times[0,1]$. The spatial resolution is $n_{x}=100, n_{y}=100$ and $n_{z}=100$. The following parameters are used in the simulation:

$$
M_{1}=M_{2}=1 \times 10^{-3}, \quad \alpha=0.001, \quad \beta=0.1, \quad \varepsilon=0.01, \quad \theta=2 \times 10^{-5}, \quad \lambda=1 .
$$

The initial conditions are taken as the randomly perturbed concentration fields as follows:

$$
\begin{aligned}
& \phi_{0}=0.2+0.001 \operatorname{rand}(x, y, z), \\
& \rho_{0}=0.3+0.001 \operatorname{rand}(x, y, z),
\end{aligned}
$$

where the rand $(x, y, z)$ is the random number in $[-1,1]$ and has zero mean. The first-order scheme LS1_tho with the time step size $\Delta t=1 \times 10^{-3}$ is implemented in this simulation. Figure 9 gives the time evolution of phase variables $\phi$ and $\rho$. As we expected, surfactants slowly migrate toward the interface with the separation of two phases.
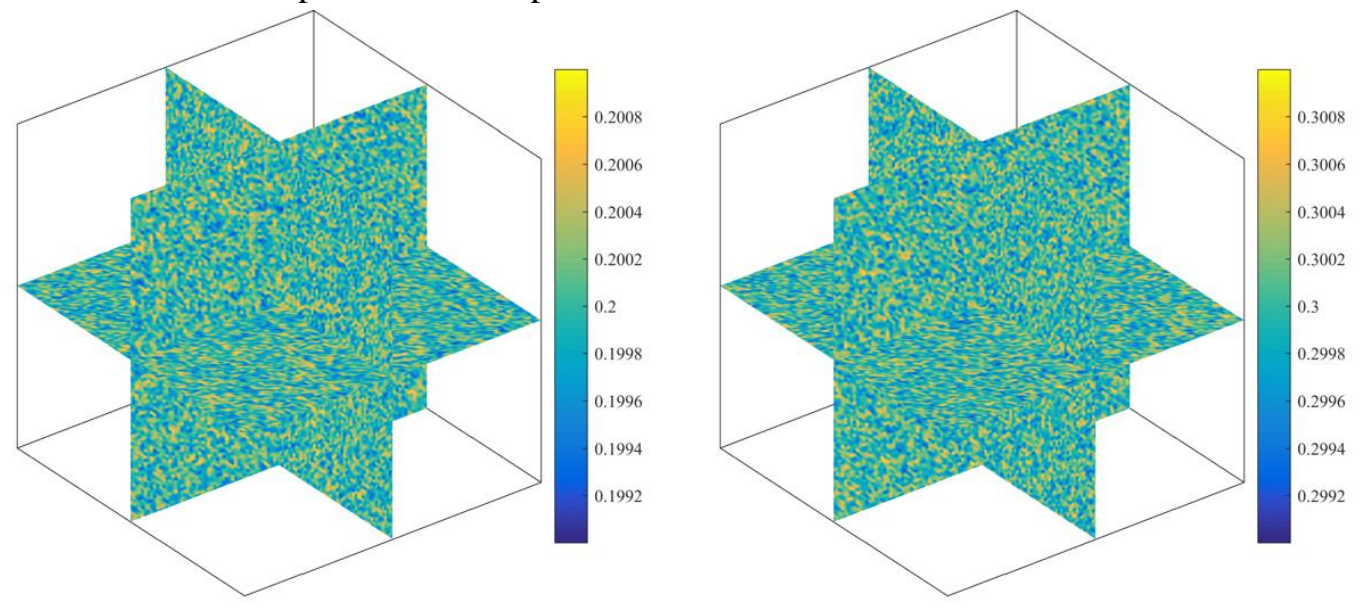

(a) $t=0$ 

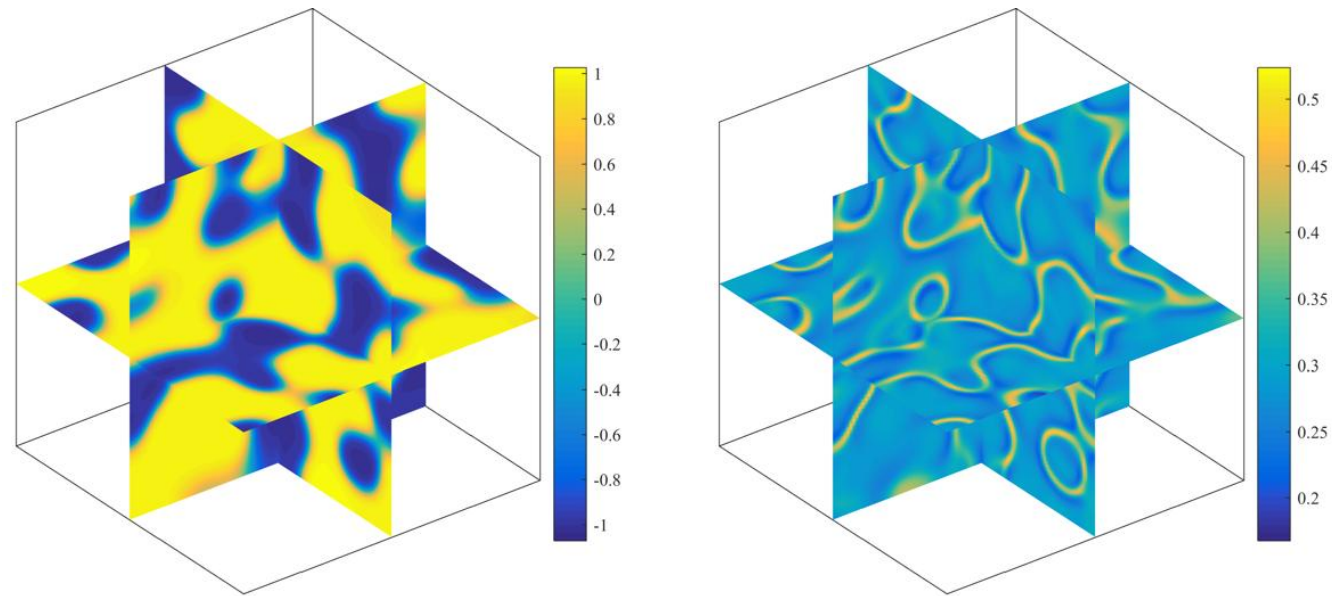

(b) $t=1$
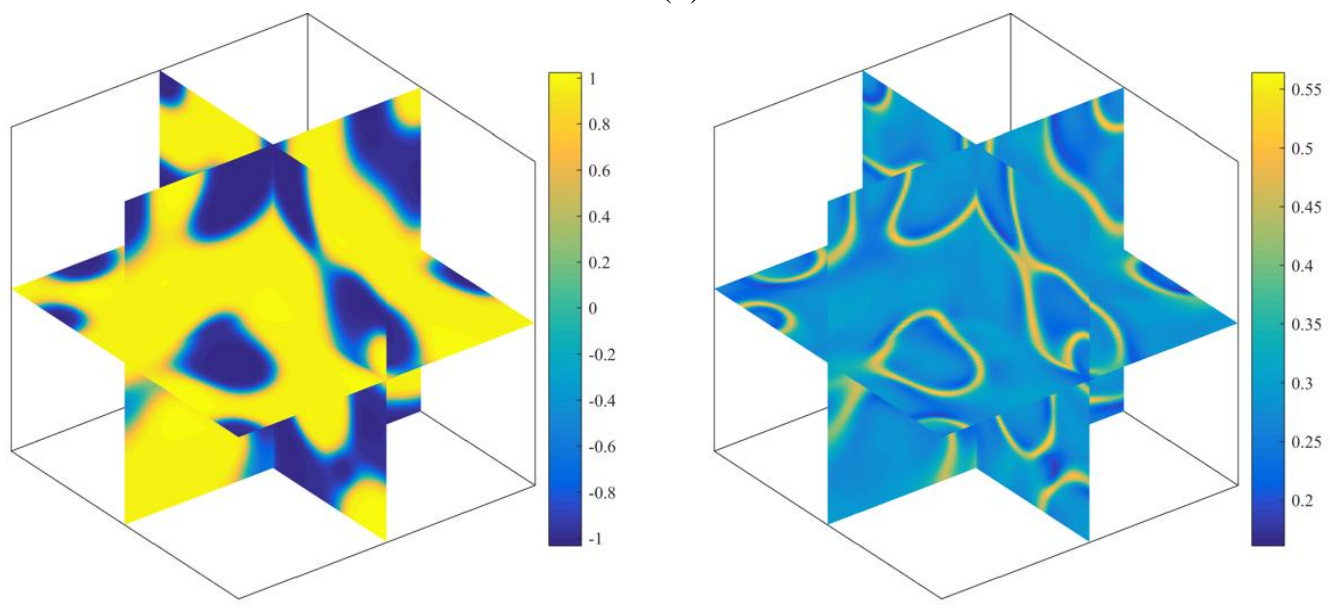

(c) $t=2.5$
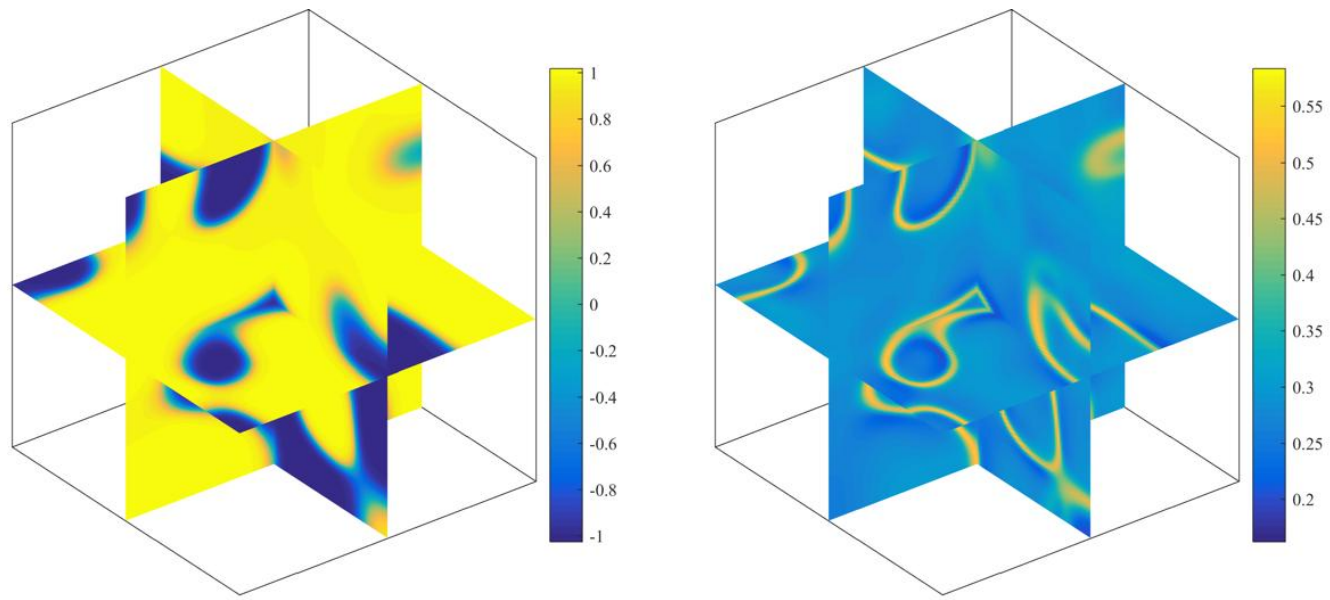

(d) $t=5$

Figure 9 Evolutions of phase variables $\phi$ and $\rho$ at different times. For each subfigure, the left is the profile of $\phi$, and the right is the profile of $\rho$. Randomly perturbed surfactants are slowly absorbed onto the interface as the two phases separated. $\left(n_{x}=n_{y}=n_{z}=100, \Delta t=1 \times 10^{-3}, M_{1}=M_{2}=1 \times 10^{-3}, \alpha=0.001, \beta=0.1, \varepsilon=0.01, \theta=2 \times 10^{-5}\right)$ 


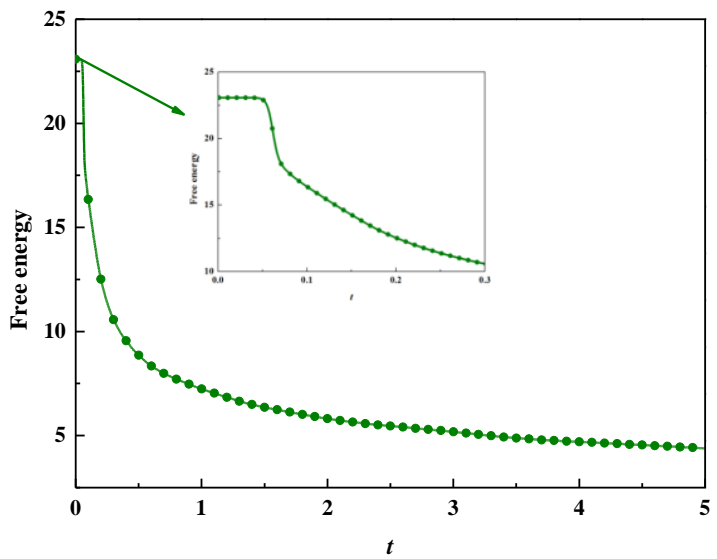

Figure 10 Time evolution of the free energy $E_{\text {tot. }}$. The trend of energy curve confirms that our scheme is unconditionally energy stable. Some important parameters and computational details can refer to the caption of Figure 9.

Again, the trend of curve in Figure 10 demonstrates the energy stability of proposed scheme. We also extract the power law for the three-dimensional coarsening dynamics, as shown in Figure 11.

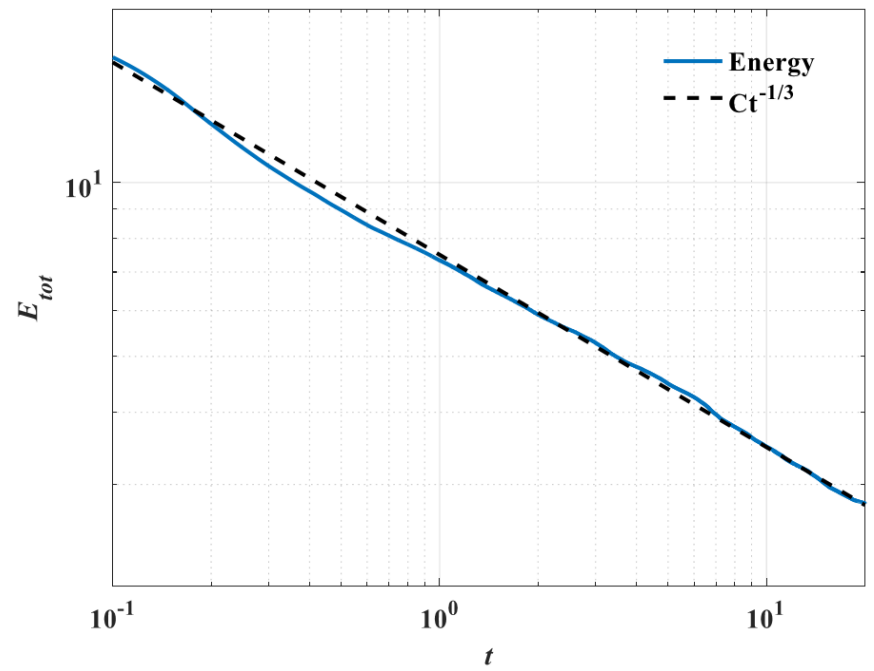

Figure 11 Time evolution of the free energy (double logarithmic scale, $t=0.1$ to 20). Free energy goes according to $E_{\text {tot }} \approx C t^{-1 / 3}(C=7.5)$ after $t=7$. Some important parameters and computational details can refer to the caption of Figure 9. To extract the power law, we have extended the simulation time to 20 .

\section{Conclusions}

In this study, we present several linear, decoupled and energy stable schemes for a phase-field surfactant model. Several scalar auxiliary variables (SAV) are introduced to transform the governing system into an equivalent form, allowing the nonlinear potentials to be treated efficiently and semi-explicitly. At each time step, the schemes involve solving only two linear elliptic differential equations, and computations of two phase field variables are totally decoupled. Moreover, the local concentration of surfactants can be obtained in a "explicit" way. The constructed schemes can also be easily extended to a large class of gradient flow problems with multiple variables or complex nonlinearities in free energy density. We further establish a rigorous proof of unconditional energy stability for the semi-implicit schemes. Numerical results in both 
two and three dimensions are obtained, which demonstrate that the accuracy, efficiency and unconditional energy stability of proposed schemes.

\section{Acknowledgement}

Jun Yao and Guangpu Zhu acknowledge that this work is supported by the National Science and Technology Major Project (2016ZX05011-001), the NSF of China (51490654, 51504276, and 51304232), and the Innovative Project of China University of Petroleum (YCX2017021). The work of Shuyu Sun and Jisheng Kou is supported by the KAUST research fund awarded to the Computational Transport Phenomena Laboratory at KAUST through the Grant BAS/1/1351-01-01.

\section{Author information}

\section{Corresponding author}

shuyu.sun@kaust.edu.sa (S. Sun). rcogfr_upc@126.com (J. Yao).

\section{First author}

b16020069@s.upc.edu.cn (G. Zhu)

\section{References}

[1] H. Garcke, K.F. Lam, B. Stinner, Diffuse interface modelling of soluble surfactants in two-phase flow, arXiv preprint arXiv:1303.2559, (2013).

[2] X. Yang, Numerical Approximations for the Cahn-Hilliard Phase Field Model of the Binary Fluid-Surfactant System, J. Sci. Comput. , (2017) 1-21.

[3] H. Liu, Y. Zhang, Phase-field modeling droplet dynamics with soluble surfactants, J. Comput. Phys. , 229 (2010) 9166-9187.

[4] S. Iglauer, Y. Wu, P. Shuler, Y. Tang, W.A. Goddard III, New surfactant classes for enhanced oil recovery and their tertiary oil recovery potential, J. Petrol. Sci. Eng., 71 (2010) 23-29.

[5] A.J. James, J. Lowengrub, A surfactant-conserving volume-of-fluid method for interfacial flows with insoluble surfactant, J. Comput. Phys., 201 (2004) 685-722.

[6] S. Khatri, A.-K. Tornberg, An embedded boundary method for soluble surfactants with interface tracking for two-phase flows, J. Comput. Phys., 256 (2014) 768-790.

[7] M. Muradoglu, G. Tryggvason, A front-tracking method for computation of interfacial flows with soluble surfactants, J. Comput. Phys. , 227 (2008) 2238-2262.

[8] Z. Zhang, S. Xu, W. Ren, Derivation of a continuum model and the energy law for moving contact lines with insoluble surfactants, Phys. Fluids, 26 (2014) 062103.

[9] M. Booty, M. Siegel, A hybrid numerical method for interfacial fluid flow with soluble surfactant, J. Comput. Phys. , 229 (2010) 3864-3883.

[10] J.-J. Xu, W. Ren, A level-set method for two-phase flows with moving contact line and insoluble surfactant, J. Comput. Phys. , 263 (2014) 71-90.

[11] D. Jacqmin, Calculation of two-phase Navier-Stokes flows using phase-field modeling, J. Comput. Phys., 155 (1999) 96-127. 
[12] J. Kou, S. Sun, Thermodynamically consistent simulation of nonisothermal diffuse-interface two-phase flow with Peng-Robinson equation of state, J. Comput. Phys. 371 (2018) 581-605.

[13] J. Kou, S. Sun, Entropy stable modeling of non-isothermal multi-component diffuse-interface two-phase flows with realistic equations of state, Comput. Methods Appl. Mech. Engrg. (2018), https://doi.org/10.1016/j.cma.2018.06.002.

[14] J. Kou, S. Sun, Thermodynamically consistent modeling and simulation of multi-component two-phase flow with partial miscibility, Comput. Method. Appl. M., 331 (2018) 623-649.

[15] J. Shen, X. Yang, Numerical approximations of allen-cahn and cahn-hilliard equations, Discrete Contin. Dyn. Syst, 28 (2010) 1669-1691.

[16] J. Shen, X. Yang, Decoupled, energy stable schemes for phase-field models of two-phase incompressible flows, SIAM J. Numer. Anal. , 53 (2015) 279-296.

[17] G. Zhu, J. Yao, A. Li, H. Sun, L. Zhang, Pore-Scale Investigation of Carbon Dioxide-Enhanced Oil Recovery, Energy Fuels, 31 (2017) 5324-5332.

[18] G. Zhu, J. Yao, L. Zhang, H. Sun, A. Li, B. Shams, Investigation of the Dynamic Contact Angle Using a Direct Numerical Simulation Method, Langmuir, 32 (2016) 11736-11744.

[19] G. Zhu, J. Kou, S. Sun, J. Yao, A. Li. Numerical approximation of a binary fluid-surfactant phase field model of two-phase incompressible flow. arXiv preprint, (2018) 1804.06305.

[20] L. Chen, J. Shen. Applications of semi-implicit Fourier-spectral method to phase field equations. Comput. Phys. Commun., 108 (1998) 147-158.

[21] P. Yue, J.J. Feng, C. Liu, J. Shen, A diffuse-interface method for simulating two-phase flows of complex fluids, J. Fluid Mech., 515 (2004) 293-317.

[22] S. Engblom, M. Do-Quang, G. Amberg, A.-K. Tornberg, On diffuse interface modeling and simulation of surfactants in two-phase fluid flow, Commun. Comput. Phys., 14 (2013) 879-915.

[23] I. Fonseca, M. Morini, V. Slastikov, Surfactants in foam stability: A phase-field model, Arch. Ration. Mech. Anal. , 183 (2007) 411-456.

[24] G. Pätzold, K. Dawson, Numerical simulation of phase separation in the presence of surfactants and hydrodynamics, Phys. Rev. E, 52 (1995) 6908.

[25] K.E. Teigen, P. Song, J. Lowengrub, A. Voigt, A diffuse-interface method for two-phase flows with soluble surfactants, J. Comput. Phys. , 230 (2011) 375-393.

[26] J. Shen, X. Yang, A phase-field model and its numerical approximation for two-phase incompressible flows with different densities and viscosities, SIAM. J. Sci. Compyt., 32 (2010) 1159-1179.

[27] G.I. Tóth, B. Kvamme, Analysis of Ginzburg-Landau-type models of surfactant-assisted liquid phase separation, Phys. Rev. E, 91 (2015) 032404.

[28] G. Zhu, H. Chen, S. Sun, J. Yao, A fully discrete energy stable scheme for a phase field moving contact line model with variable densities and viscosities, arXiv preprint arXiv:1801.08739, (2018).

[29] M. Laradji, H. Guo, M. Grant, M.J. Zuckermann, The effect of surfactants on the dynamics of phase separation, J. Phys.: Condens. Matter 4(1992) 6715.

[30] G. Gompper and M. Schick. Self assembling amphiphilic systems, volume 16 of phase transitions and critical phenomena. Academic Press, London, 1994.

[31] Y. Li, J. Kim, A comparison study of phase-field models for an immiscible binary mixture with surfactant, The European Physical Journal B, 85 (2012) 340.

[32] R. van der Sman, M. Meinders. Analysis of improved Lattice Boltzmann phase field method for soluble surfactants[J]. Comput. Phys. Commun, 199 (2016) 12-21. 
[33] S. Komura, H. Kodama, Two-order-parameter model for an oil-water-surfactant system, Phys. Rev. E, 55 (1997) 1722.

[34] O. Theissen, G. Gompper, Lattice-Boltzmann study of spontaneous emulsification, The European Physical Journal B-Condensed Matter and Complex Systems, 11 (1999) 91-100.

[35] R. Van der Sman, S. Van der Graaf, Diffuse interface model of surfactant adsorption onto flat and droplet interfaces, Rheol. Acta 46 (2006) 3-11.

[36] C.-H. Teng, I.-L. Chern, M.-C. Lai, Simulating binary fluid-surfactant dynamics by a phase field model, Discrete and Continuous Dynamical Systems-Series B, Special issue for FAN2010 in honor of J. Thomas Beale, in press, (2012).

[37] X. Yang, L. Ju, Linear and unconditionally energy stable schemes for the binary fluid-surfactant phase field model, Computer Methods in Applied Mechanics and Engineering, 318 (2017) 1005-1029.

[38] S. Gu, H. Zhang, Z. Zhang, An energy-stable finite-difference scheme for the binary fluid-surfactant system, J. Comput. Phys. , 270 (2014) 416-431.

[39] X. Yang, L. Ju, Efficient linear schemes with unconditional energy stability for the phase field elastic bending energy model, Comput. Method. Appl. M., 315 (2017) 691-712.

[40] X. Yang, J. Zhao, Q. Wang, J. Shen, Numerical approximations for a three-component Cahn-Hilliard phase-field model based on the invariant energy quadratization method, Mathematical Models and Methods in Applied Sciences, 27 (2017) 1993-2030.

[41] J. Shen, J. Xu, J. Yang, The scalar auxiliary variable (SAV) approach for gradient flows, J. Comput. Phys. , 353 (2018) 407-416.

[42] J. Shen, J. Xu, J. Yang, A new class of efficient and robust energy stable schemes for gradient flows, arXiv preprint arXiv:1710.01331, (2017).

[43] J. Kou, S. Sun, X. Wang, Linearly decoupled energy-stable numerical methods for multi-component two-phase compressible flow, arXiv preprint arXiv:1712.02222, (2017).

[44] A. Yun, Y. Li, J. Kim, A new phase-field model for a water-oil-surfactant system, Applied Mathematics and Computation, 229 (2014) 422-432.

[45] M. Copetti, C.M. Elliott, Numerical analysis of the Cahn-Hilliard equation with a logarithmic free energy, Numerische Mathematik, 63 (1992) 39-65.

[46] S. Aland S, A. Voigt. Benchmark computations of diffuse interface models for two - dimensional bubble dynamics. Int. J. Number. Meth. Fl., 69(2012) 747-761.

[47] M. Gao, X. Wang. An efficient scheme for a phase field model for the moving contact line problem with variable density and viscosity. J. Comput. Phys., 272(2014) 704-718.

[48] H. Garcke, B. Niethammer, M. Rumpf, U. Weikard. Transient coarsening behaviour in the Cahn-Hilliard model. Acta Materialia, 51(2003) 2823-2830. 